\title{
UNIQUENESS OF POSITIVE SOLUTIONS OF THE HEAT EQUATION
}

\author{
HAROLD DONNELLY
}

\begin{abstract}
Uniqueness is proved for positive solutions of the heat equation on complete Riemannian manifolds with Ricci curvature bounded from below.
\end{abstract}

1. Introduction. Let $M$ be a complete Riemannian manifold with Ricci curvature bounded from below. The Laplacian of $M$, acting on functions, will be denoted by $\Delta$. The associated heat equation problem is

$$
\begin{gathered}
(\partial / \partial t-\Delta) u(x, t)=0 \\
u(x, 0)=f(x) .
\end{gathered}
$$

We assume that $u(x, t)$ is a continuous function on $M \times[0, \infty)$.

There have been several recent results on uniqueness for $u$ lying in various function spaces. In [3], Dodziuk proved uniqueness for bounded and continuous $u$. Strichartz [10] proved $L^{p}$ uniqueness, $1<p<\infty$. Karp and $\mathrm{Li}[5]$ gave a unified proof of these results by working in appropriate weighted $L^{p}$-spaces.

In this paper, we consider positive solutions of the heat equation. Let $K(x, y, t)$ be the fundamental solution. Our main result is

THEOREM 1.1. If $u(x, t)$ is any nonnegative solution of the heat equation, then

$$
u(x, t)=\int_{M} K(x, y, t) f(y) d y .
$$

In particular, the integral converges and $u$ is uniquely determined by its initial data $f$.

If $M$ is the real line, then Theorem 1.1 is due to Widder [11]. We follow the outline of his proof. However, an explicit formula for $K(x, y, t)$ is no longer available. One must use appropriate estimates instead. In particular, this provides an interesting application of the lower bound of the heat kernel, an estimate of Cheeger and Yau [1].

A different proof for uniqueness of positive solutions has been obtained independently by $\mathrm{Li}$ and Yau [8]. For manifolds of bounded geometry, one may consult Koranyi and Taylor [6].

2. Reduction to zero initial data. Let $K(x, y, t)$ be the fundamental solution of the heat equation, as in [3]. Then $K(x, y, t)$ is the positive solution obtained by taking a $\delta$ measure, at $y$, as initial data. Suppose that $u(x, t)$ is any nonnegative

Received by the editors February 3, 1986.

1980 Mathematics Subject Classification (1985 Revision). Primary 53C99, 58G05. 
solution of the heat equation. One has

LEMMA 2.1. $u(x, t) \geq \int_{M} K(x, y, t) u(y, 0) d y$. In particular, the integral converges.

Proof. Let $D_{i}$ be an exhaustion of $M$ by relatively compact domains. Suppose that $\phi_{i}$ is a nonnegative continuous function of compact support, which is equal to one on $D_{i}$. Set $\tilde{u}_{i}(x, t)=\int_{M} K(x, y, t) \phi_{i}(y) u(y, 0) d y$. Then $\tilde{u}_{i}$ satisfies the heat equation since the integral has compact support. Also, $\tilde{u}_{i}(x, t)$ vanishes at infinity, for fixed $t$, since the heat semigroup preserves the bounded continuous functions' vanishing at infinity [3, p. 713]. Applying the maximum principle of $[\mathbf{3}, \mathrm{p}$. 705] to the compact domains $D_{j}, j>i$, we obtain $u(x, t)-\tilde{u}_{i}(x, t) \geq-\varepsilon_{j}$, for $x \in D_{j}$. Since $\tilde{u}_{i}$ vanishes at infinity, $\varepsilon_{j} \rightarrow 0$ as $j \rightarrow \infty$. This gives $u(x, t) \geq \tilde{u}_{i}(x, t)$ for $x \in M$. Recalling the definition of $\tilde{u}_{i}$ and applying the monotone convergence theorem gives Lemma 2.1.

We introduce the notation $\tilde{u}(x, t)=\int_{M} K(x, y, t) u(y, 0) d y$. Lemma 2.1 states that $u \geq \tilde{u}$. We will eventually prove equality. One first observes

LEMMA 2.2. $\tilde{u}(x, t)$ satisfies the heat equation. Moreover, $\tilde{u}(x, t)$ is continuous and has initial values $u(x, 0)$.

PROOF. The functions $\tilde{u}_{i}$ form a nondecreasing sequence of solutions to the heat equation. Moreover, the local $L^{1}$-norms of $\tilde{u}_{i}(x, t), 0<t_{1}<t<t_{2}$, are uniformly bounded since $\tilde{u}_{i}(x, t) \leq u(x, t)$. Therefore, one may apply the convergence criterion of $[3$, p. 711$]$. This proves that $\tilde{u}$ satisfies the heat equation and is continuous on $M \times(0, \infty)$. Alternatively, we could directly apply classical interior estimates [4], instead of $[3$, p. 711].

It remains to check that $\tilde{u}$ has the required initial values. Suppose that $D$ is a sufficiently small relatively compact domain containing $x$. Then

$$
u(x, 0)=\lim _{t \rightarrow 0} u(x, t) \geq \lim _{t \rightarrow 0} \tilde{u}(x, t)
$$

and

$$
\lim _{t \rightarrow 0} \tilde{u}(x, t)=\lim _{t \rightarrow 0} \int_{M} K(x, y, t) u(y, 0) d y \geq \lim _{t \rightarrow 0} \int_{D} K(x, y, t) u(y, 0) d y
$$

However, by the local asymptotic expansion of the heat kernel [1, p. 468]:

$$
\lim _{t \rightarrow 0} \int_{D} K(x, y, t) u(y, 0) d y=u(x, 0)
$$

Combining the above inequalities gives $u(x, 0)=\lim _{t \rightarrow 0} \tilde{u}(x, t)$. The proof of Lemma 2.2 is complete.

In summary, $w(x, t)=u(x, t)-\tilde{u}(x, t)$ is a nonnegative solution of the heat equation with zero initial data.

3. Uniqueness of positive solutions. Let $w(x, t)$ be a nonnegative solution of the heat equation with $w(x, 0)=0$. We need to show that $w(x, t)=0$. Define $v(x, t)=\int_{0}^{t} w(x, s) d s$. Clearly, it suffices to show that $v$ vanishes identically, since $w$ is nonnegative. One begins by observing, as in [11]: 
LEMMA 3.1. $v_{t}=\Delta v=w$. In particular, $v$ is nonnegative, satisfies the heat equation, and is subharmonic in $x$.

ProOF. Obviously, $v_{t}=w$, by the fundamental theorem of calculus. Also

$$
\Delta v=\int_{0}^{t} \Delta w(x, s) d s=\int_{0}^{t} w_{s}(x, s) d s=w(x, t)-w(x, 0)=w(x, t) .
$$

The differentiation under the integral is justified by local regularity theorems for parabolic equations $[\mathbf{4}$, p. 75$]$.

We now obtain a growth estimate for $v(x, t)$. Suppose that $r(p, x)$ is the geodesic distance from a fixed basepoint $p$ in $M$. One has

LEMMA 3.2. For any $\varepsilon>0$ and $0 \leq t \leq \varepsilon$ we may write

$$
v(x, t) \leq C_{1} \exp \left(C_{2} r^{2}(p, x)\right)
$$

The constants $C_{1}$ and $C_{2}$ are independent of $t$.

PROOF. Let $B$ denote the ball centered at $x$ and having radius $r(p, x)+1$. Suppose that $T>0$ is arbitrary. Lemma 2.1 gives:

$$
v(p, t+T) \geq \int_{M} K(p, y, T) v(y, t) d y \geq \int_{B} K(p, y, T) v(y, t) d y .
$$

The main result of $[\mathbf{1}]$ is a lower bound for the heat kernel,

$$
K(p, y, T) \geq C_{3} \exp \left(-C_{4} r^{2}(p, y)\right) .
$$

However, $y \in B$, so from the triangle inequality $r(p, y) \leq 2 r(p, x)+1$. Substitution yields

$$
\int_{B} v(y, t) d y \leq C_{5} \exp \left(C_{6} r^{2}(p, x)\right) v(p, t+T) .
$$

The mean value estimate of [7], applied to the nonnegative subharmonic function $v$, gives

$$
v(x, t) \leq C_{7} \exp \left(C_{8} r(p, x)\right) \int_{B} v(y, t) d y .
$$

Combining the last two inequalities yields

$$
v(x, t) \leq C_{9} \exp \left(C_{10} r^{2}(p, x)\right) v(p, t+T) .
$$

As $t$ varies over the interval $0 \leq t \leq \varepsilon$, the quantity $v(p, t+T)$ remains uniformly bounded in $t$. This proves Proposition 3.2.

To complete the proof of Theorem 1.1, we recall the following.

PROPOSITION 3.3. Let $v(x, t)$ be any solution of the heat equation, for $(x, t) \in$ $M \times[0, \varepsilon]$, which satisfies

$$
|v(x, t)| \leq C_{1} e^{C_{2} r^{2}(p, x)}
$$

for some $C_{1}$ and $C_{2}$. If $v(x, 0)=0$, then $v$ is identically zero.

ProOF. This follows from the method of [2, pp. 1038-1039]. For additional details, and generalizations to weighted $L^{p}$-spaces, the reader may consult [5].

By Lemma 3.2 and Proposition 3.3, one has that $v$ is identically zero. Thus $w=u-\tilde{u}$ is identically zero. Recalling the definition of $\tilde{u}$, we have $u(x, t)=$ $\int_{M} K(x, y, t) f(y) d y$, where $u(y, 0)=f(y)$. This completes the proof of Theorem 1.1. 


\section{REFERENCES}

1. J. Cheeger and S. T. Yau, A lower bound for the heat kernel, Comm. Pure Appl. Math. 34 (1981), 465-480.

2. S. Y. Cheng, P. Li, and S. T. Yau, On the upper estimate of the heat kernel of a complete Riemannian manifold, Amer. J. Math. 103 (1981), 1021-1063.

3. J. Dodziuk, Maximum principle for parabolic inequalities and the heat flow on open manifolds, Indiana Univ. Math. J. 32 (1983), 703-716.

4. A. Friedman, Partial differential equations of parabolic type, Prentice Hall, Englewood Cliffs, N. J., 1964.

5. L. Karp and P. Li, The heat equation on complete Riemannian manifolds, preprint.

6. A. Koranyi and J. C. Taylor, Miminal solutions of the heat equation and uniqueness of the positive Cauchy problem on homogeneous spaces, Proc. Amer. Math. Soc. 94 (1985), 273-278.

7. $\mathrm{P} . \mathrm{Li}$ and $\mathrm{R}$. Schoen, $L^{p}$ and mean value properties of subharmonic functions on Riemannian manifolds, Acta Math. 153 (1984), 279-302.

8. P. Li and S. T. Yau,, On the parabolic kernel of the Schrödinger operator, Acta Math. 156 (1986), 153-202.

9. H. Royden, Real analysis, Macmillan, New York, 1968.

10. R. Strichartz, Analysis of the Laplacian on a complete riemannian manifold, J. Funct. Anal. 52 (1983), 48-79.

11. D. V. Widder, The heat equation, Academic Press, New York, San Francisco, and London, 1975.

Department of Mathematics, Purdue University, West Lafayette, Indiana 47907 